\title{
Preoperative prediction of the pathological stage of advanced gastric cancer by 18 F-fluoro-2-deoxyglucose positron emission tomography
}

\section{Kota Yamada}

Kobe University Graduate School of Medicine

\section{Naoki Urakawa}

Kobe University Graduate School of Medicine

Shingo Kanaji ( $\nabla$ kanashin@med.kobe-u.ac.jp )

Kobe University Graduate School of Medicine

Hiroshi Hasegawa

Kobe University Graduate School of Medicine

Masashi Yamamoto

Kobe University Graduate School of Medicine

Kimihiro Yamashita

Kobe University Graduate School of Medicine

Takeru Matsuda

Kobe University Graduate School of Medicine

Taro Oshikiri

Kobe University Graduate School of Medicine

\section{Satoshi Suzuki}

Kobe University Graduate School of Medicine

Yoshihiro Kakeji

Kobe University Graduate School of Medicine

\section{Research Article}

Keywords: fluorodeoxyglucose F18, positron emission tomography computed tomography, stomach neoplasm, lymphatic metastasis

Posted Date: February 25th, 2022

DOI: https://doi.org/10.21203/rs.3.rs-1379694/v1

License: (c) (i) This work is licensed under a Creative Commons Attribution 4.0 International License. Read Full License 


\section{Abstract}

In recent years, the usefulness of neoadjuvant chemotherapy for resectable advanced gastric cancer, particularly stage III, has been reported. Preoperative staging is mainly determined by computed tomography (CT), and the usefulness of ${ }^{18} \mathrm{~F}-$ fluoro-2-deoxyglucose positron emission tomography/CT (FDG-PET/CT) for gastric cancer remains unclear. The study aimed to evaluate the usefulness of FDG-PET/CT in preoperative diagnosis of advanced gastric cancer. We retrospectively enrolled 113 patients with gastric cancer who underwent preoperative FDG-PET/CT. All patients underwent gastrectomy with lymph-node dissection. The maximum standardized uptake value (SUVmax) of the primary tumor (TSUVmax) and lymph nodes (N-SUVmax) were measured for all patients. The cutoff values of T-SUVmax for pathological T3/4 from receiver operating characteristic analysis were 8.28 for differentiated and 4.32 for undifferentiated types. The T-SUVmax and N-SUVmax cutoff values for pathological lymph-node metastasis were 4.32 and 1.82, respectively. Multivariate analysis showed that T-SUVmax for differentiated types was a significant predictor of pathological T3/4, and N-SUVmax was a significant predictor of lymph-node metastasis. In conclusion, the SUVmax of FDG-PET/CT was a useful predictor of pathological T3/4 and lymph-node metastasis in gastric cancer. The diagnosis by preoperative FDG$\mathrm{PET} / \mathrm{CT}$ allowed a more accurate staging of gastric cancer than by CT scan alone.

\section{Introduction}

Gastric cancer (GC) is one of the most common malignancies and the third leading cause of cancer-related deaths worldwide [1]. Although diagnosis, surgical techniques, and adjuvant therapies for gastric cancer have advanced over the years, the prognosis for stage III and IV GC remains poor [2]. In recent years, neoadjuvant chemotherapy (NAC) has become the first choice for advanced GC due to the advantages of early chemotherapy for micro-metastasis, good general condition, and high treatment compliance in Western countries $[3,4]$. Similarly, a clinical study of NAC for GC with advanced lymph-node metastasis is currently underway by the Japan Clinical Oncology Group (JCOG) $[5,6]$. One of the problems of NAC therapy is the uncertainty of preoperative diagnosis of GC, which leads to unnecessary treatment and excessive invasion for early stage patients. Currently, the preoperative diagnosis of GC is mainly performed by esophagogastroduodenoscopy, endoscopic ultrasonography, and computed tomography (CT), but accuracy remains inadequate [7-9]. Thus, other useful predictors of advanced GC are essential for selection of an appropriate preoperative treatment.

${ }^{18} \mathrm{~F}$-fluoro-2-deoxyglucose positron emission tomography/CT (FDG-PET/CT) is a valuable tool for diagnosis, staging, and identification of recurrence in many malignancies $[10,11]$. However, the role of preoperative FDG-PET/CT in GC remains unclear. In this study, we measured the maximum standardized uptake values (SUVmax) of primary tumors and lymph nodes on FDG- PET/CT and assessed their usefulness as predictors of advanced GC.

\section{Results}

\section{1 | Patients' characteristics}

The patients' characteristics are shown in Table $1.0 f 113$ patients, pathologically, 60 patients (53.1\%) were the differentiated and 53 (46.9\%) were the undifferentiated type. Fifty-nine (52.2\%) patients were clinically diagnosed as T3/4 by esophagogastroduodenoscopy and CT. Fifty-eight (51.3\%) patients were clinically diagnosed as positive for lymph-node metastasis on the basis of the lymph-node diameters measured by CT. Pathological T3/4 was confirmed in $56(49.6 \%)$ patients and lymph-node metastasis in $54(47.8 \%)$ patients.

\section{2 | Relationship between histological type and SUVmax}


The median T-SUVmax and N-SUVmax were 5.08 (range, 0.78-34.1) and 1.57 (range, 0.74-14.3), respectively. We evaluated the relationship between histological type and SUVmax. The median T-SUVmax was 6.34 (range, 1.18-34.1) for the differentiated type and 3.57 (range, 0.78-21.6) for the undifferentiated type. The median N-SUVmax was 1.52 (range, 0.74-12.3) for the differentiated type and 1.67 (range, 0.89-14.3) for the undifferentiated type. The T-SUVmax were higher in the differentiated type than in the undifferentiated type $(P=0.02)$, but there was no significant difference in the N-SUVmax $(P=0.21)$.

\section{3 | Cutoff SUVmax for diagnosis of pathological T3/4 tumor and lymph-node metastasis}

The AUCs of the T-SUVmax values to predict pT3/4 determined by ROC curve analysis were $0.86(95 \% \mathrm{Cl}, 0.76-0.95)$ for the differentiated type and $0.80(0.69-0.92)$ for the undifferentiated type (Figure 1a, b). The AUCs of the T-SUVmax and N-SUVmax for predicting pathological lymph-node metastasis were $0.66(0.56-0.76)$ and $0.76(0.67-0.85)$, respectively (Figure 1c). The cutoff values of T-SUVmax for pT3/4 were 8.28 for the differentiated type and 4.32 for the undifferentiated type, and the cutoff values of T-SUVmax and N-SUVmax for pathological lymph-node metastasis were 4.32 and 1.82 , respectively.

\section{4 | Usefulness of T-SUVmax for diagnosis of the tumor invasion depth}

The results of the univariate and multivariate logistic regression analysis for pT3/4 are shown in Table 2 . In the differentiated type, univariate logistic regression showed that high preoperative CA19-9, cT3/4, cN positive, and high TSUVmax ( $\geq 8.28$ ) were significantly associated with pT3/4. In the multivariate analysis, cT3/4 (Odds ratio [OR], 62.1; 95\% $\mathrm{Cl}, 5.32-724.0 ; P<0.01)$, high T-SUVmax (OR, 8.88; 95\% Cl, 1.35-58.3; $P=0.02)$ were significantly predictive factors for pT3/4, but in the undifferentiated type, cT3/4 and high T-SUVmax $(\geq 4.32)$ were not.

\section{5 | Usefulness of N-SUVmax for diagnosis of lymph-node metastasis}

Univariate logistic regression revealed that Male, cT3/4, cN positive, high T-SUVmax ( $\geq 4.32$ ), and high N-SUVmax $(\geq 1.82)$ were significantly associated with lymph-node metastasis. In the multivariate analysis, Male $(\mathrm{OR}, 4.36 ; 95 \% \mathrm{Cl}$, 1.43-13.3; $P<0.01$ ), cT3/4 (OR, 4.10; 95\% Cl, 1.31-12.9; $P=0.02)$, and high N-SUVmax (OR, 16.5; 95\% Cl, 3.84-70.5; $P<$ 0.01 ) were significant predictors of lymph-node metastasis (Table 3). To evaluate the diagnostic ability of CT and FDGPET/CT, we compared their sensitivity, specificity, positive predictive value (PPV), negative predictive value (NPV), and accuracy (Table 4). Although the sensitivity, specificity, PPV, and NPV for diagnosing lymph-node metastasis based on lymph-node diameters by CT were $68.5 \%, 69.5 \%, 67.3 \%$, and $70.7 \%$, respectively, the sensitivity, specificity, PPV, and NPV for diagnosing lymph-node metastasis based on N-SUVmax by FDG-PET/CT were $53.7 \%, 94.9 \%, 90.6 \%$, and $69.1 \%$, respectively. The accuracy of diagnosis of lymph-node metastasis was higher by FDG-PET/CT than by CT $(75.2 \%$ vs. $69.0 \%)$.

\section{Discussion}

The SUVmax in primary GC tumors has been shown to correlate with tumor invasion depth [12-14], but few studies have predicted pT status by using a cutoff value. By analyzing the relationship between histological type and SUVmax, we found that the uptake of FDG in primary tumors of GC depended on histological type, with higher uptake in differentiated types and lower uptake in undifferentiated types. Therefore, in undifferentiated types, multivariate analysis did not show that the T-SUVmax was useful for diagnosis of pT3/4. This finding is consistent with those of several previous reports and explains the low utility of FDG-PET/CT in GC [15-17]. However, our results showed that the T-SUVmax was a significant predictor of $\mathrm{pT} 3 / 4$ diagnosis in differentiated types by using a cutoff value with the ROC curve.

In the diagnosis of lymph-node metastasis, Oh et al. have shown that T-SUVmax on FDG-PET/CT is a useful predictor of pathological lymph-node metastasis in GC [18]. These studies focused only on the metabolic activity of the primary 
tumor. We considered that using the metabolic activity of lymph nodes as a predictor of lymph-node metastasis would further improve the diagnostic accuracy. Tsunoda et al. revealed that in rectal cancer, a high N-SUVmax (>1.5) was more useful in preoperative diagnosis of lymph-node metastasis than was the lymph-node diameter [19]. Our study showed similar results in GC, and N-SUVmax was a useful predictor for diagnosing pathological lymph-node metastasis regardless of histological type.

Fukagawa et al. reported that it was difficult to accurately diagnose pathological lymph-node metastasis and reported the sensitivity and specificity of CT without FDG-PET/CT were $62.5 \%$ and $65.7 \%$, respectively [20]. In our analysis, the accuracy of CT alone in diagnosing pathological lymph-node metastasis was similar to their results, but the combination of diagnosis by FDG-PET/CT improved the accuracy of preoperative diagnosis of pathological lymph-node metastasis. Even in cases diagnosed as positive for lymph-node metastasis by CT, if N-SUVmax was low in FDG-PET/CT, 94\% $(17 / 18)$ of the cases were negative for pathological lymph-node metastasis, which is also useful for diagnosis of negative prediction.

Considering the cost of FDG-PET/CT, it may not be practical to perform it preoperatively for all patients with GC. Therefore, it is important to select patients with comorbidities who are expected to experience more adverse events from NAC treatment, and patients with poor renal function who cannot undergo contrast-enhanced CT. The use of FDGPET/CT combined with conventional diagnostic methods, such as esophagogastroduodenoscopy and CT, can avoid unnecessary NAC treatment for such patients and contribute to appropriate treatment selection.

This study had several limitations. First, this was a single-institution retrospective study, so selection bias was possible. However, FDG-PET/CT is expensive, and it is valuable to demonstrate the significance of this study in a small number of cases. Second, the SUV of small-sized primary tumors and lymph nodes could have been underestimated due to partial volume effects.

In conclusion, the diagnosis of the pathological stage of advanced GC by preoperative FDG-PET/CT was shown to be useful for selecting an appropriate treatment strategy.

\section{Patients And Methods}

\section{1 | Patients}

From January 2014 to December 2019, 113 patients who had undergone gastrectomy for adenocarcinoma of GC at Kobe University Hospital were analyzed retrospectively in this study. These patients had been diagnosed with gastric adenocarcinoma by esophagogastroduodenoscopy and had undergone preoperative CT and FDG-PET/CT for initial staging. Preoperative diagnoses of the depth of tumor invasion (T status) and lymph-node metastasis ( $\mathrm{N}$ status) were diagnosed by esophagogastroduodenoscopy and CT. For evaluation of $\mathrm{N}$ status, a size $\geq 8 \mathrm{~mm}$ measured by CT was considered positive with reference to the JCOG 1302A study [20]. All patients received distal or total gastrectomy with D1+ or D2 lymph-node dissection. All lymph nodes suspected to be metastatic on preoperative CT or FDG-PET/CT were dissected in surgery. Patients who received any preoperative treatments, such as chemotherapy or radiotherapy, were excluded. The diagnosis had been confirmed by histopathological examination according to the $15^{\text {th }}$ edition of the Japanese Classification of Gastric Carcinoma [21]. Pathologically, papillary and well- and moderately differentiated adenocarcinomas are defined as differentiated, whereas poorly differentiated, mucinous, and signet-ring-cell adenocarcinomas are defined as undifferentiated. This study was approved by the Ethics Committee of Kobe University (No. B210017).

\section{2 | FDG-PET/CT protocol}


Whole-body FDG-PET scans were performed on a PET scanner (Philips Allegro, Philips Medical System, Best, the Netherlands). All patients fasted for $\geq 6$ hours before injection of 222-333 MBq (6-9 mCi) of FDG and had blood glucose levels of $<160 \mathrm{mg} / \mathrm{dl}$ at the time of injection. Approximately 1 hour after IV administration of 222 to $333 \mathrm{MBq}$ (6$9 \mathrm{mCi}$ ) of FDG, a static emission scan was performed. Emission PET scans were reconstructed by using the row-action maximum-likelihood algorithm resulting in a $128 \times 128$ matrix. After the PET scans, the patients underwent CT scans performed at $120 \mathrm{kV}$ and $80 \mathrm{~mA}$. All PET and CT images were integrated by using automatic image-fusion software (Syntegra; SUN Microsystems). The T-SUVmax and N-SUVmax for all patients were measured by FDG-PET/CT (Figure 2).

\section{3 | Statistical analyses}

Statistical analysis was performed to determine the relationships between clinicopathological parameters, including the SUVmax, and pathological T3/4 (pT3/4) tumor and lymph-node metastasis by using the Mann-Whitney U-test for continuous variables and Fisher's exact test for categorical variables, as appropriate. All $P$ values $<0.05$ were considered to be indicative of statistical significance. A multiple logistic regression model was used to identify useful predictors for diagnosis of pT3/T4 tumors and lymph-node metastasis. Covariates found to be significant in the univariate analysis at $P<0.05$ were included in the multivariate model. Receiver operating characteristic (ROC) analysis was performed to assess the confidence of the SUVmax for predicting PT3/T4 tumors and lymph-node metastasis, and the areas under the curves (AUCs) were measured. The optimal cutoff value was determined by ROC analysis. All statistical analyses were performed with EZR [22] (Saitama Medical Center, Jichi Medical University, Saitama, Japan), which is a graphical user interface for $\mathrm{R}$ (The R Foundation for Statistical Computing, Vienna, Austria). More precisely, it is a modified version of $\mathrm{R}$ commander designed to add statistical functions frequently used in biostatistics.

\section{Declarations}

\section{ACKNOWLEDGMENTS}

We would like to express our appreciation to Dr Yuichi Wakabayashi, Division of Radiation Oncology, Kobe University Hospital, for his advice.

We would also like to thank Enago (www.enago.jp) for the English language review.

Conflict of Interest: The authors declare that they have no conflict of interest.

Funding: The authors did not receive any grants, equipment, or funding for this study

Human rights statement and informed consent: All procedures followed were in accordance with the ethical standards of the responsible committee on human experimentation (institutional and national) and with the Helsinki declaration of 1964 and later versions. Informed consent to be included in the study, or the equivalent, was obtained from all patients.

\section{Data Availability}

The datasets generated and/or analyzed during the current study are available from the corresponding author on reasonable request.

\section{References}

1. Bray F, Ferlay J, Soerjomataram I, Siegel RL, Torre LA, Jemal A. Global cancer statistics 2018: GLOBOCAN estimates of incidence and mortality worldwide for 36 cancers in 185 countries. CA Cancer J Clin. 68, 394-424 (2018). 
2. Nashimoto A, et al. Gastric cancer treated in 2002 in Japan: 2009 annual report of the JGCA nationwide registry. Gastric Cancer. 16, 1-27 (2013).

3. Al-Batran SE, et al. Perioperative chemotherapy with fluorouracil plus leucovorin, oxaliplatin, and docetaxel versus fluorouracil or capecitabine plus cisplatin and epirubicin for locally advanced, resectable gastric or gastrooesophageal junction adenocarcinoma (FLOT4): A randomised, phase 2/3 trial. Lancet. 393, 1948-57 (2019).

4. Cunningham $D$, et al. Perioperative chemotherapy versus surgery alone for resectable gastroesophageal cancer. $N$ Engl J Med. 355, 11-20 (2006).

5. Tokunaga $\mathrm{M}$, et al. Phase III trial to evaluate the efficacy of neoadjuvant chemotherapy with S-1 plus oxaliplatin followed by D2 gastrectomy with adjuvant S-1 in locally advanced gastric cancer: Japan Clinical Oncology Group study JCOG1509 (NAGISA trial). J Clin Oncol. 35 (2017).

6. Kataoka K, et al. A randomized Phase II trial of systemic chemotherapy with and without trastuzumab followed by surgery in HER2-positive advanced gastric or esophagogastric junction adenocarcinoma with extensive lymph node metastasis: Japan Clinical Oncology Group study JCOG1301 (Trigger Study). Jpn J Clin Oncol. 45, 1082-6 (2015).

7. Kim SH, et al. Preoperative $\mathrm{N}$ staging of gastric cancer by stomach protocol computed tomography. J Gastric Cancer. 13, 149-56 (2013).

8. Tsujimoto H, Sugasawa H, Ono S, Ichikura T, Yamamoto J, Hase K. Has the accuracy of preoperative diagnosis improved in cases of early-stage gastric cancer? World J Surg. 34, 1840-6 (2010).

9. Kwee RM, Kwee TC. Imaging in assessing lymph node status in gastric cancer. Gastric Cancer. 12, 6-22 (2009).

10. Ulaner GA. PET/CT for patients with breast cancer: Where is the clinical impact? AJR Am J Roentgenol. 213, 254-65 (2019).

11. Fletcher JW, et al. Recommendations on the use of 18F-FDG PET in oncology. J Nucl Med. 49, 480-508 (2008).

12. Namikawa T, Okabayshi T, Nogami M, Ogawa Y, Kobayashi M, Hanazaki K. Assessment of (18)F-fluorodeoxyglucose positron emission tomography combined with computed tomography in the preoperative management of patients with gastric cancer. Int J Clin Oncol. 19, 649-55 (2014).

13. Kudou M, et al. Value of preoperative PET-CT in the prediction of pathological stage of gastric cancer. Ann Surg Oncol. 25, 1633-9 (2018).

14. Yoon JK, et al. Clinicopathologic parameters associated with the FDG-avidity in staging of early gastric cancer using 18F-FDG PET. Medicine. 98, e16690 (2019).

15. Shimada H, Okazumi S, Koyama M, Murakami K. Japanese Gastric Cancer Association Task Force for Research Promotion: Clinical utility of ${ }^{1} \otimes \mathrm{F}$-fluoro-2-deoxyglucose positron emission tomography in gastric cancer. A systematic review of the literature. Gastric Cancer. 14, 13-21 (2011).

16. Maman A, Sahin A, Ayan AK. The relationship of SUV value in PET-CT with tumor differentiation and tumor markers in gastric cancer. Eurasian J Med. 52, 67-72 (2020).

17. Choi BH, Song HS, An YS, Han SU, Kim JH, Yoon JK. Relation between fluorodeoxyglucose uptake and glucose Transporter-1 expression in gastric signet ring cell carcinoma. Nucl Med Mol Imaging. 45, 30-5 (2011).

18. Oh HH, et al. The peak-standardized uptake value (P-SUV) by preoperative positron emission tomography-computed tomography (PET-CT) is a useful indicator of lymph node metastasis in gastric cancer. J Surg Oncol. 104, 530-3 (2011).

19. Tsunoda Y, Ito M, Fujii H, Kuwano H, Saito N. Preoperative diagnosis of lymph node metastases of colorectal cancer by FDG-PET/CT. Jpn J Clin Oncol. 38, 347-53 (2008).

20. Fukagawa $T$, et al. A prospective multi-institutional validity study to evaluate the accuracy of clinical diagnosis of pathological stage III gastric cancer (JCOG1302A). Gastric Cancer. 21, 68-73 (2018). 
21. Japanese Gastric Cancer Association. Japanese Classification of Gastric Carcinoma. The 15th edition (in Japanese). Tokyo: Kanehara (2017).

22. Kanda Y. Investigation of the freely available easy-to-use software 'EZR' for medical statistics. Bone Marrow Transplant. 48, 452-8 (2013).

\section{Tables}

Table 1: Patient characteristics and surgical procedure 


$$
\text { Sex }
$$

Male

77

Female

36

Tumor location

$\begin{array}{ll}\text { Upper } & 26 \\ \text { Middle } & 54 \\ \text { Lower } & 33\end{array}$

Histological type

Papillary 1

Well- and moderately differentiated $\quad 59$

Poorly differentiated 43

Signet ring cell $\quad 8$

Mucinous 2

cT status

T2

54

T3

47

T4

12

$\mathrm{cN}$ status

NO

55

N1

42

N2

14

N3

2

cStage

प

36

口

40

]

34

प

3

pT status 
$\mathrm{pN}$ status

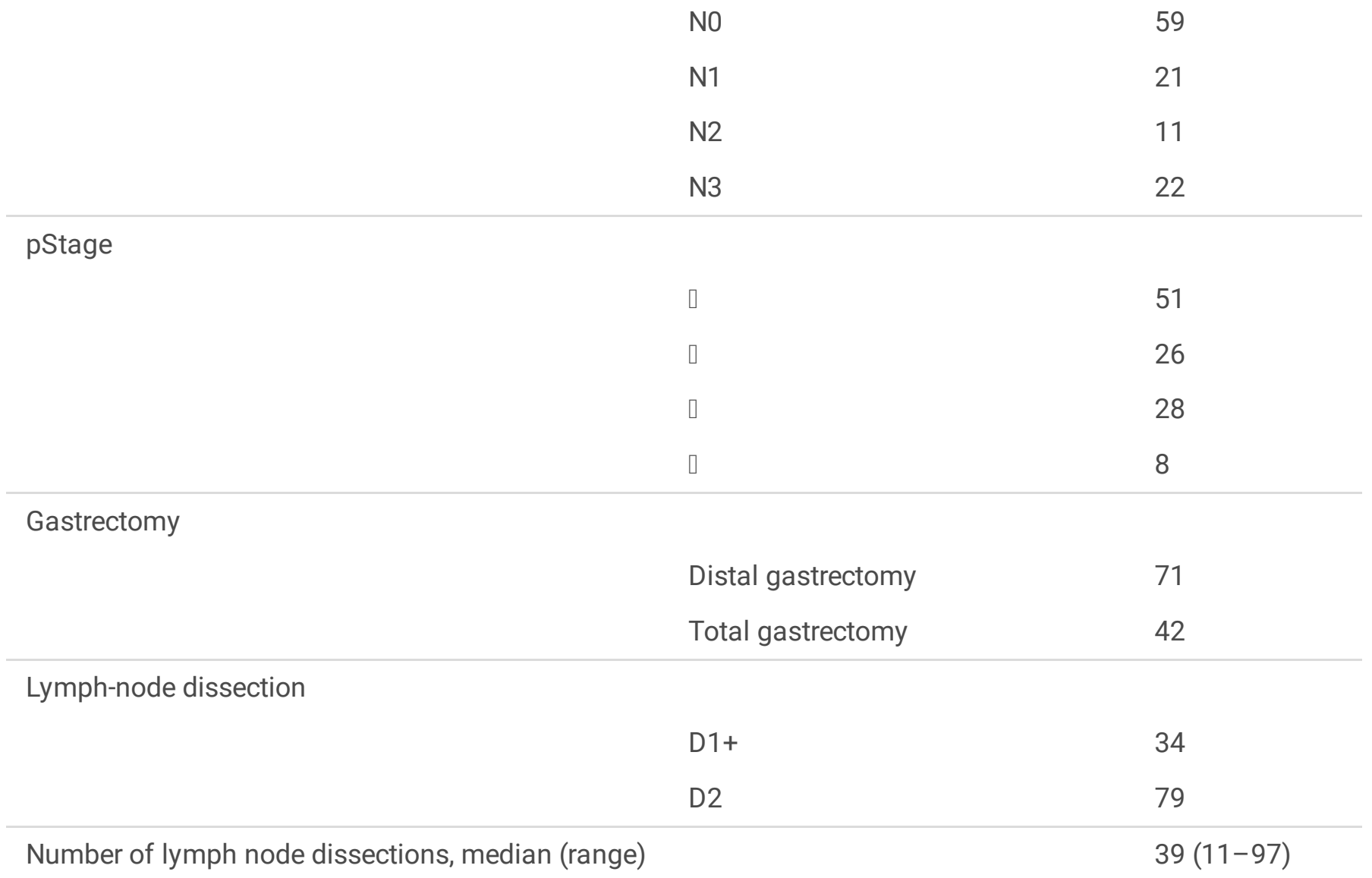

Table 2: Univariate and multivariate analysis for pathological T3/T4 tumors 


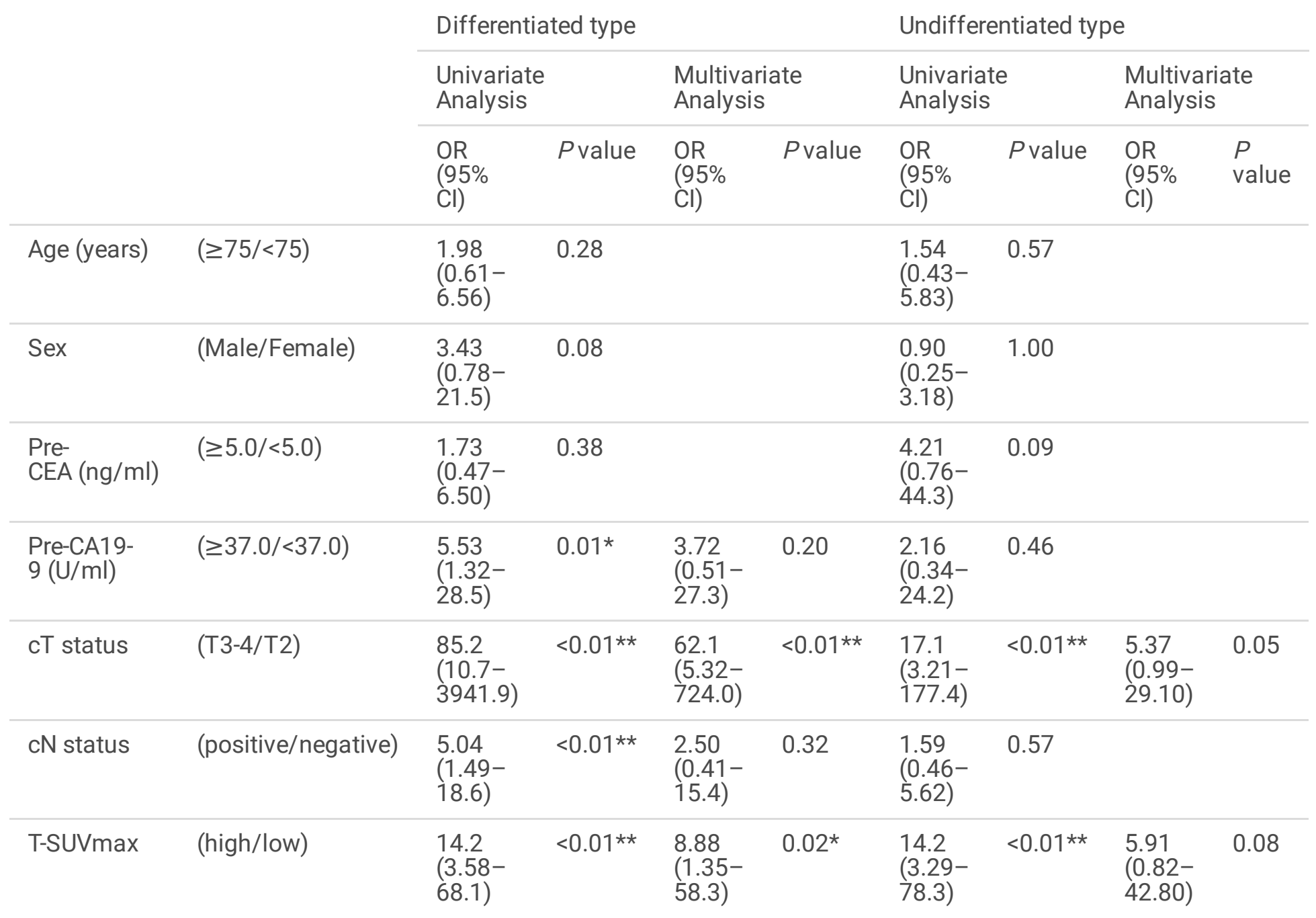

$\mathrm{OR}=$ Odds Ratio, $\mathrm{Cl}=$ Confidence Interval, pre = preoperative, SUV max = Maximum standardized uptake value

* $P$ value $<0.05$, ** $P$ value $<0.01$

Table 3: Univariate and multivariate analysis for pathological lymph-node metastasis 


\begin{tabular}{|c|c|c|c|c|c|}
\hline & & \multicolumn{2}{|l|}{ Univariate Analysis } & \multicolumn{2}{|c|}{ Multivariate Analysis } \\
\hline & & OR $(95 \% \quad \mathrm{Cl})$ & $P$ value & OR $(95 \%$ & $P$ value \\
\hline Age (years) & $(\geq 75 /<75)$ & $1.44(0.64-3.31)$ & 0.44 & & \\
\hline Sex & (Male/Female) & $3.43(1.37-9.14)$ & $<0.01^{\star *}$ & $4.36(1.43-13.3)$ & $<0.01^{\star *}$ \\
\hline \multirow[t]{2}{*}{ Histology } & (undifferentiated/ & $1.46(0.65-3.29)$ & 0.35 & & \\
\hline & differentiated) & & & & \\
\hline Pre-CEA (ng/ml) & $(\geq 5.0 /<5.0)$ & $0.93(0.36-2.39)$ & 1.00 & & \\
\hline Pre-CA19-9 (U/ml) & $(\geq 37.0 /<37.0)$ & $1.75(0.62-5.15)$ & 0.34 & & \\
\hline cT status & $(\mathrm{T} 3-4 / \mathrm{T} 2)$ & $4.24(1.82-10.26)$ & $<0.01^{\star \star}$ & $4.10(1.31-12.9)$ & $0.02 *$ \\
\hline cN status & (positive/negative) & $4.88(2.08-11.9)$ & $<0.01^{\star *}$ & $2.03(0.75-5.50)$ & 0.16 \\
\hline T-SUV max & $(\geq 4.32 /<4.32)$ & $2.74(1.20-6.4)$ & $0.01 *$ & $0.58(0.18-1.85)$ & 0.36 \\
\hline N-SUV max & $(\geq 1.82 /<1.82)$ & $21.0(5.73-118.2)$ & $<0.01$ ** & $16.5(3.84-70.5)$ & $<0.01 * *$ \\
\hline
\end{tabular}

$\mathrm{OR}=$ Odds Ratio; $\mathrm{Cl}$ = Confidence Interval, pre = preoperative, SUV max = Maximum standardized uptake value

* $P$ value $<0.05$, ** $P$ value $<0.01$

Table 4: Diagnostic ability of CT scan and FDG-PET/CT to detect pathological lymph-node metastasis

\begin{tabular}{|c|c|c|c|c|c|}
\hline & $\begin{array}{l}\text { Sensitivity } \\
(\%)\end{array}$ & $\begin{array}{l}\text { Specificity } \\
(\%)\end{array}$ & $\begin{array}{l}\text { Positive predictive } \\
\text { value }(\%)\end{array}$ & $\begin{array}{l}\text { Negative predictive } \\
\text { value (\%) }\end{array}$ & $\begin{array}{l}\text { Accuracy } \\
(\%)\end{array}$ \\
\hline CT scan & 68.5 & 69.5 & 67.3 & 70.7 & 69.0 \\
\hline \multicolumn{6}{|l|}{$\begin{array}{l}\text { (lymph-node } \\
\text { diameter } \geq 8 \mathrm{~mm} \text { ) }\end{array}$} \\
\hline \multicolumn{6}{|l|}{ FDG-PET/CT } \\
\hline$(\mathrm{N}-S U V \max \geq 1.82)$ & & & & & \\
\hline $\begin{array}{l}\text { CT scan and FDG- } \\
\text { PET/CT }\end{array}$ & 50.0 & 98.3 & 96.4 & 68.2 & 75.2 \\
\hline
\end{tabular}

SUV max = Maximum standardized uptake value

\section{Figures}


a

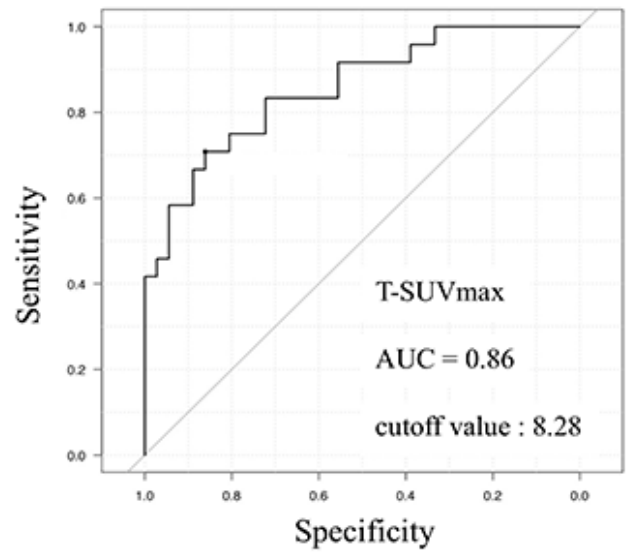

b

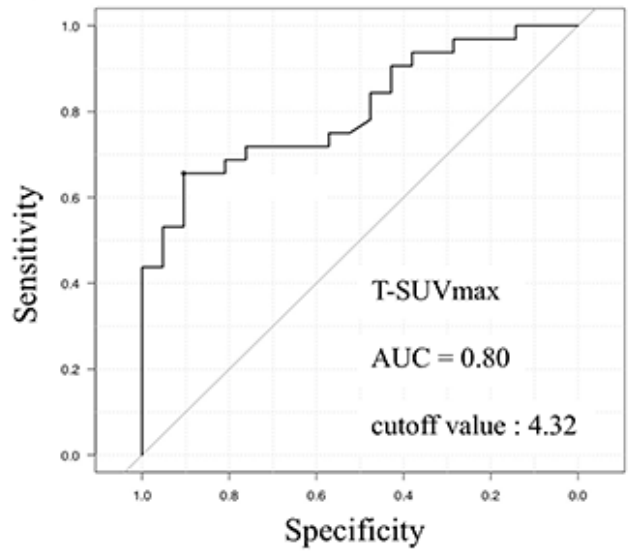

c

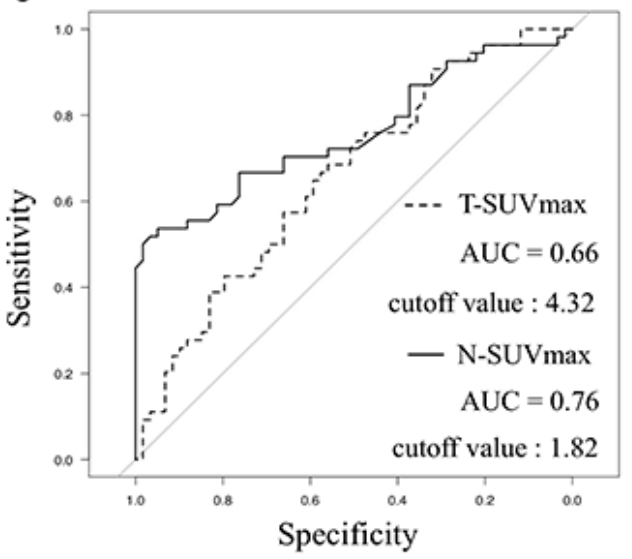

\section{Figure 1}

a,b: Receiver operating characteristic (ROC) curve for assessment of the confidence of T-SUVmax to predict pathological T3 or T4 tumor. (a: differentiated type, b: undifferentiated type)

C: ROC curve for assessment of the confidence of T-SUVmax and N-SUVmax to predict pathological lymph-node metastasis.

\section{Figure 2}

Preoperative FDG-PET/CT of a 72-year-old man showing FDG uptake (a: T-SUVmax, 11.53; b: N-SUVmax, 12.29). This patient underwent subtotal gastrectomy and stage III gastric cancer was confirmed (pT3N2M0 pStage『A, welldifferentiated tubular adenocarcinoma). 